\title{
Ca II H sunspot tomography from the photosphere to the chromosphere
}

\author{
V. M. J. Henriques ${ }^{1,2}$ and D. Kiselman ${ }^{1,2}$ \\ ${ }^{1}$ Institute for Solar Physics, Royal Swedish Academy of Sciences, Albanova University Center, 10691 Stockholm, Sweden \\ 2 Stockholm Observatory, Dept. of Astronomy, Stockholm University, Albanova University Center, 10691 Stockholm, Sweden \\ e-mail: vasco@astro.su.se
}

Received 5 April 2013 / Accepted 6 July 2013

\section{ABSTRACT}

\begin{abstract}
Aims. We aim at gaining insight into the thermal properties of different small-scale structures related to sunspots. Methods. We use filtergrams in the Ca II H filter at the Swedish 1-m Solar Telescope to study the relationship between fine structure at different heights in a sunspot.

Results. The methods for destretching and aligning the different image data work well. The magnetic spine structure in the outer parts of the sunspot penumbra is found to be associated with higher intensities in the Ca II $\mathrm{H}$ wing passbands but with less steep vertical temperature gradients. Dark lanes in a light bridge behave very similarly to dark cores in penumbral filaments. Fibril structures are seen in the line-core images over the umbra and penumbra.

Conclusions. The observations add support to the idea that penumbral filaments, light bridges, and umbral dots are caused by similar processes of overturning convection. Observations in the Ca II H \& K wings are a promising observable, complementing others, for testing simulation results for sunspots at high spatial resolution.
\end{abstract}

Key words. sunspots - Sun: surface magnetism - Sun: magnetic topology - Sun: faculae, plages - Sun: chromosphere techniques: high angular resolution

\section{Introduction}

The formation heights of the $\mathrm{Ca}$ II $\mathrm{H}$ and $\mathrm{K}$ line profiles span from the continuum-forming layers of the solar photosphere to possibly even higher than $\mathrm{H} \alpha$ (Vernazza et al. 1981). Over a large fraction of this range, the lines are formed in local thermodynamic equilibrium, Ca II is the majority species of calcium, and most of the $\mathrm{Ca}$ II is in the ground state. These lines thus offer an opportunity to extract temperature as a function of height in the solar atmosphere. Shine \& Linsky (1974), Rouppe van der Voort (2002), Sheminova et al. (2005), Sheminova (2012), and Beck et al. (2013) took advantage of this using high-resolution spectra. In an earlier paper (Henriques 2012), we generalised the technique of Shine \& Linsky (1974) for use with Ca II H filtergrams at different locations in the line wing, which were acquired by tilting a $0.11-\mathrm{nm}$ interference filter so as to tune the transmission profile. The resulting images can be used to derive temperatures at different depths, which can then be differentiated to produce maps showing the vertical temperature gradient. The aim of the current paper is to employ this technique to investigate a sunspot by comparing maps showing temperature gradients, intensity passbands from different heights, and total circular polarisation (TCP) in the Fe I $630 \mathrm{~nm}$ line pair, which acts as a proxy for the longitudinal magnetic field.

Taking advantage of the high-resolution Ca II line-core data, we also take a look at the filamentary structures above the penumbra and umbra. The existence of fine structure in the umbra was inferred indirectly by Socas-Navarro et al. (2000), Centeno et al. (2005), and Tritschler et al. (2008). Filamentary structures were observed by Socas-Navarro et al. (2009) in the $\mathrm{Ca}$ II $\mathrm{H}$ line core using Hinode and again more recently at higher resolution ( $\left.0{ }^{\prime} 18\right)$ by de la Cruz Rodríguez et al. (2013). Socas-Navarro et al. (2009) showed that these seem to have a surprisingly large horizontal extent. In this paper we take advantage of the high resolution of our data to take a new look at such structures at $0 . ' 10$.

\section{Observations and data reduction}

The observations were performed with the Swedish 1-m Solar Telescope (SST, Scharmer et al. 2003) on 23 May 2010. The target was active region NOAA 11072, including a sunspot observed at $\mu=\cos \theta=0.97$. Using a standard setup, the beam was split in a blue and a red part.

The setup, observations, and reduction of the blue-beam data are described in detail by Henriques (2012). Likewise, the redbeam data and their reduction are described in Scharmer \& Henriques (2012), with some differences detailed below, where we otherwise summarise the main aspects of the reductions.

The passbands of the blue cameras are listed in Table 1. Two of the cameras were mounted behind a wideband (WB) filter in the pseudo continuum between the $\mathrm{Ca}$ II $\mathrm{H}$ and $\mathrm{K}$ lines. The third camera was mounted behind a $0.12 \mathrm{~nm}$ wide filter at $396.47 \mathrm{~nm}$ in the $\mathrm{Ca}$ II $\mathrm{H}$ blue wing. This will be referred to as the fixedwing filter (FW). The fourth camera was placed behind a narrowband interference filter mounted on a rotation stage with central wavelength just red-wise of the core of $\mathrm{Ca}$ II $\mathrm{H}$. The rotation stage allows tilting of the filter with respect to the optical axis, which shifts the centre of the passband towards the blue, effectively scanning the $\mathrm{Ca}$ II $\mathrm{H}$ blue wing as described by Löfdahl et al. (2011).

The red-beam data were acquired with the CRisp Imaging SPectropolarimeter (CRISP, Scharmer 2006; Scharmer et al. 2008). The polarisation signal was corrected for telescope polarisation using the time-dependent telescope model of 
Table 1. Properties of the blue data used.

\begin{tabular}{lcccccc}
\hline \hline & Description & $\begin{array}{c}\text { Tilt } \\
\text { angle }\end{array}$ & $\begin{array}{c}\lambda_{\mathrm{c}} \\
{[\mathrm{nm}]}\end{array}$ & $\begin{array}{c}F W H M \\
{[\mathrm{~nm}]}\end{array}$ & $\begin{array}{c}m_{i} \\
{\left[\mathrm{~g} \mathrm{~cm}^{-2}\right]}\end{array}$ & $\lg \tau_{500}$ \\
\hline LC & Tiltable (1) & 1.2 & 396.84 & 0.11 & 0.17 & -2.62 \\
TF2 & Tiltable (2) & 2.5 & 396.74 & 0.14 & 0.90 & -1.31 \\
FW & Fixed wing & - & 396.47 & 0.12 & 2.49 & -0.59 \\
TF5 & Tiltable (5) & 4.2 & 396.47 & 0.19 & 2.61 & -0.42 \\
TF7 & Tiltable (7) & 6.4 & 395.93 & 0.28 & 3.56 & -0.27 \\
WB & Wideband & - & 395.37 & 1.00 & & \\
\hline
\end{tabular}

Notes. Columns: designation used in the text, description, tilt angle filter central wavelength, full width at half maximum, average effective formation depth in column mass as determined in Henriques (2012), and the equivalent optical depth in HolMul. The derived quantities (DIF, G57, TCP) are described in the text.

Selbing (2010). The difference to the treatment in Scharmer $\&$ Henriques (2012) is that here we use the data from the Fe I $630.25 \mathrm{~nm}$ line in addition to the Fe I $630.1 \mathrm{~nm}$ line. Furthermore, we process the data to match the blue images and do not perform cross-talk or cavity-error corrections since we only intend to use integrated Stokes $V$ spectra.

Both data sets are almost simultaneous single scans selected for quality using the RMS contrast of the raw red-beam frames. The red scan required $16.5 \mathrm{~s}$ and overlaps in time with the blue scan, which lasted $9.7 \mathrm{~s}$. Image reconstruction was done with the MOMFBD code (van Noort et al. 2005) using non-simultaneous object compensation as described in Henriques (2012).

The scale is $0.034(25 \mathrm{~km})$ per pixel for the blue data and 0. '059 $(43 \mathrm{~km})$ per pixel for the red data. The images from the red beam were scaled (using cubic interpolation), aligned (with a slight rotation), and then destretched (as in Shine et al. 1994) to the blue data using the respective WB images as reference. The subfields of the plage and the sunspot region were destretched individually to reduce any potential errors from fieldof-view dependent geometrical distortions from either setup. The destretch in the umbra fails in some areas leaving distortions, so we avoid interpreting the Stokes $V$ signal in the umbra.

Two differential quantities are computed from the blue data. The first is the vertical temperature gradient $\frac{\Delta T}{\Delta m}$. It is designated as G57 in Henriques (2012) and computed from tilted-filter positions TF5 and TF7 (see Table 1) using an implementation of the Shine \& Linsky (1974) method to assign temperatures and depth values to each pixel. The G57 map is computed for a depth in column mass of $m=3.09 \mathrm{~g} \mathrm{~cm}^{-2}$.

The G57 maps spatial resolution is limited to 0 !' 17 , which is lower than the other blue data because of the effect of the pupil apodisation on the point spread function (PSF) at large filter-tilt angles (Löfdahl et al. 2011; Henriques 2012). The spatial resolution of the red-beam data is about 0 .' 16 , while that of the other blue data approaches $0{ }^{\prime} 10$. To make use of the higher resolution in the differential maps, we also employ a proxy of the deep temperature gradient in the shape of the scaled difference between the intensities of the FW and the WB passbands:

$\mathrm{DIF}=I_{\mathrm{WB}}-I_{\mathrm{FW}} \frac{\left\langle I_{\mathrm{WB}}\right\rangle}{\left\langle I_{\mathrm{FW}}\right\rangle}$.

This measure, which is very similar to one of the proxies studied in Leenaarts et al. (2006), gives maps that show a close resemblance to G57 but at the full resolution allowed by the data $(0.1)$.

Finally, we compute the total circular polarisation,

$\mathrm{TCP}=\int\left|S_{V}\right| \mathrm{d} \lambda$ integrating over both the 630.1 and $630.2 \mathrm{~nm}$ line profiles (similarly to Bellot Rubio et al. 2007). We use this as a proxy of the longitudinal magnetic field. The TCP is not sensitive to Doppler shifts but will be dependent on temperature and temperature gradients.

Figure 1 shows maps of all observables except the near linecore passband TF2. We note that in some of the other figures we show the negative of the gradient measures G57 and DIF to make visual comparisons easier.

\section{Relation between vertical temperature gradients and total circular polarization}

Before discussing the sunspot, we show in Fig. 2 four maps of a region just outside the spot, near its vertex. This is the same field as displayed in Fig. 8 of Henriques (2012), and it contains granulation, ribbons of bright points, and a small pore. The gradient measures, G57 and DIF, are displayed as negative images to make a comparison with the TCP map easier. This means that regions with a flat vertical temperature gradient are bright and those with a steep gradient are dark in those maps. We note first that the G57 map looks similar to the proxy measure DIF, but with lower resolution and more noise. This gives some justification for using DIF as a temperature-gradient indicator with the full spatial resolution of the data.

There is a close correspondence between the TCP map and the two negative gradient maps. The notable exception is the non-magnetic intergranular lanes. They show up in the temperature-gradient maps as bright structures (flat gradients) but naturally are not visible in the TCP map, being nonmagnetic. Thus Fig. 2 illustrates at very high spatial resolution the flat temperature gradients in magnetic regions. The nonmagnetic regions show the expected pattern of steep gradients in granules and flatter gradients in the intergranular lanes.

We now turn to the penumbra to investigate the correlation between TCP and temperature gradients there. Figure 3 consists of colour-coded maps of TCP that are multiplied with a binary mask selecting the penumbra and then with one of two different masks constructed from the G57 gradient maps. In the left column this gradient mask selects flat-gradient areas (low G57 values). The right-column images use a binary mask with high G57 values and thus display regions where the vertical temperature gradient is steep.

The upper row of Fig. 3 uses TCP without any spatial filtering. It is clear that the left-hand image shows more yellow and red colours than the right-hand image, meaning that there is an inverse correlation between TCP and G57 so that the lowgradient mask picks up more of the high-TCP regions. These high-TCP areas correspond to magnetic spines (following the description of Lites et al. 1993) with magnetic fields that are stronger and/or more vertical than in the surrounding penumbra. In the same way, the low-TCP, or inter-spine, regions are associated with steeper temperature gradients. There are, however, global patterns muddling this inverse correlation: a radial TCP gradient with TCP decreasing outwards in the penumbra, a centre-side/limb-side asymmetry, and a dependence of the TCP signal on intensity. To partly remove these trends, in the lower image pair the TCP is normalised with continuum intensity and an unsharp mask is applied. The resulting maps show more clearly that magnetic spines in the mid and outer penumbra are associated with relatively flat vertical temperature gradients. At the umbral/penumbral border this relation breaks down with most penumbral structures showing high TCP values - though 
V. M. J. Henriques and D. Kiselman: Ca II H sunspot tomography from the photosphere to the chromosphere

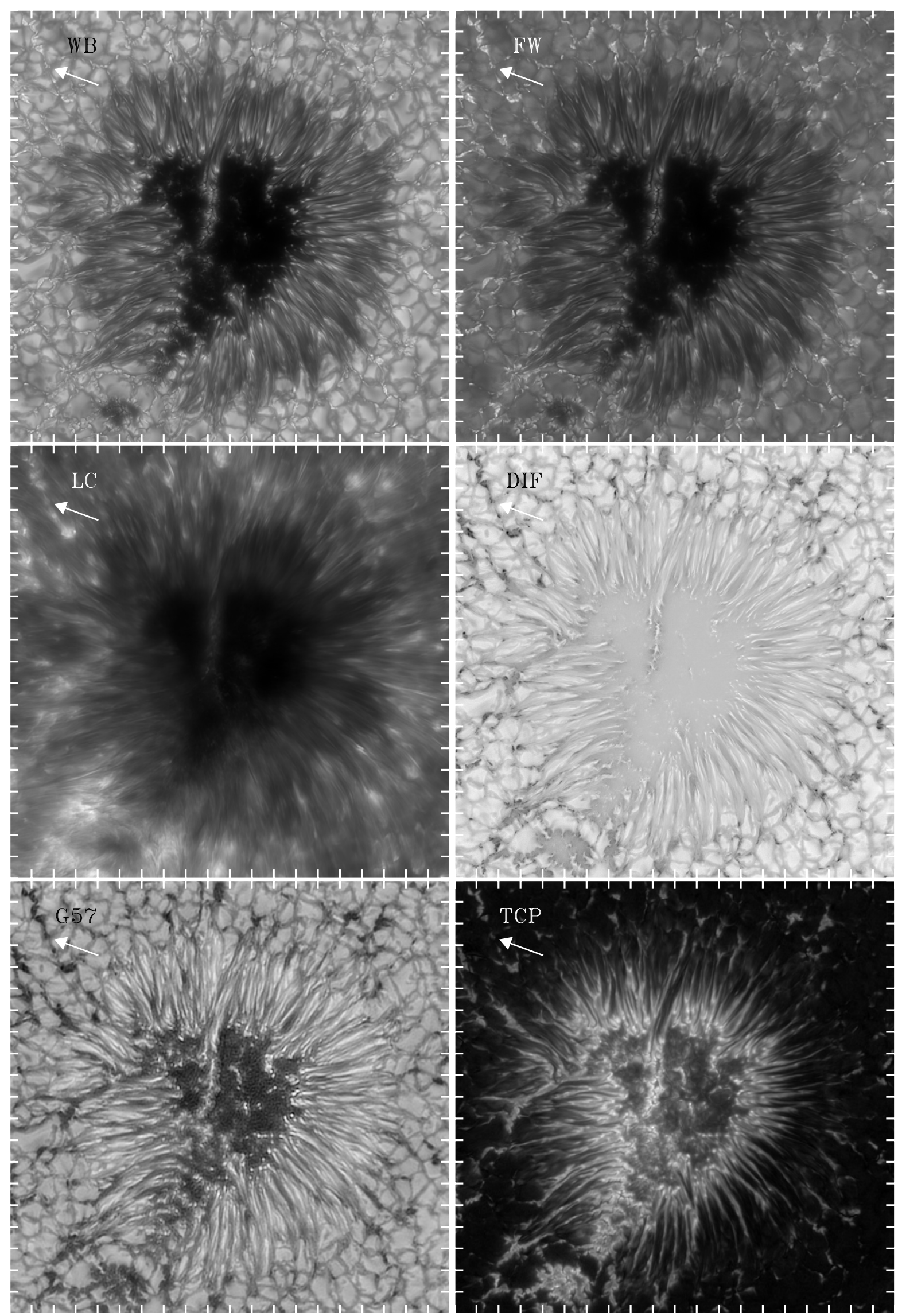

Fig. 1. Maps of observables described in Table 1 and derived quantities (DIF, G57, TCP). The arrow points towards the solar disk centre. Tickmarks have a spacing of $2^{\prime \prime}$. Field of view is $40 \times 40^{\prime \prime}$. 

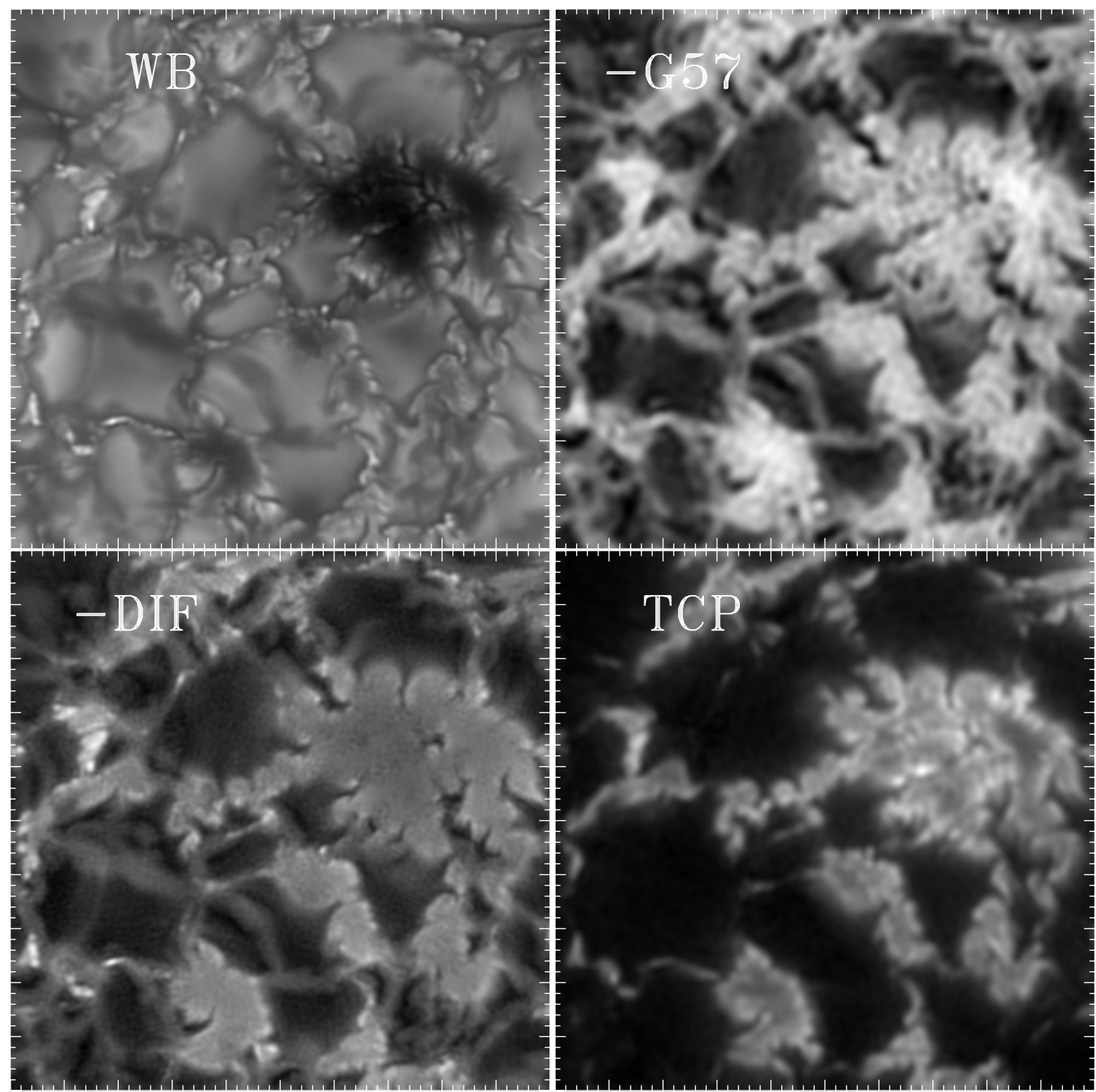

Fig. 2. Plage area close to the vertex of the sunspot. From top to bottom, left to right: WB, -G57, -DIF, and TCP. Major tickmarks at 1". Field of view is $10 \times 10^{\prime \prime}$

at the scale shown this could be due to mixing with an umbral signal.

We remind ourselves that TCP is a problematic diagnostic since it is dependent on both temperature and temperature gradient. However, the relation found here is the opposite of the expectation that regions with high temperatures and steep gradients should show larger TCP because of the stronger line profiles there. The data instead show that steep temperature gradients, as measured in the outer wings of $\mathrm{Ca}$ II $\mathrm{H}$, are associated with low values of TCP.

Figure 4 shows a portion of the limb-side penumbra in three filter passbands with a range of formation heights, the gradient measures DIF and G57, and TCP. As in Fig. 2, the gradient maps are shown as negative images in order to ease inter-comparisons. This means that regions with steep vertical temperature gradients show up as dark features in these two maps.

All images have been overlaid with a single contour calculated from the TCP map and selected in order to to outline the spine structure. We first note that there is some correlation between the intensity maps and the TCP map, although the matches are not perfect. The intensity is higher in the spines. This is a visual confirmation of Table 8 in Scharmer et al. (2011, SOM). The passband that seems to show the spines most clearly is TF2. The gradient maps also show correspondence with the intensities such that many features are discernible in both. However, this last relation is sometimes reversed. Whereas the bright peripheral penumbral grains at the umbral border display steep gradients (dark in the negative image), the spines show bright features with flat temperature gradients. This means that in the inner penumbra the intensity-gradient correlation is positive and thus granulation-like, while it becomes reversed further out.

Still in Fig. 4, the DIF and G57 gradient maps correlate well with the spine pattern seen in TCP: the spines tend to have flat temperature gradients. This is a detailed illustration of the global result from Fig. 3. It is also obvious how the relation breaks down in the inner penumbra since the heads of penumbral filaments (peripheral penumbral grains) which show a strong TCP signal, show strong positive signals in the G57 and DIF maps (a steep temperature gradient shows up dark in the images). Some of these heads are the extremity of dark-cored filaments 

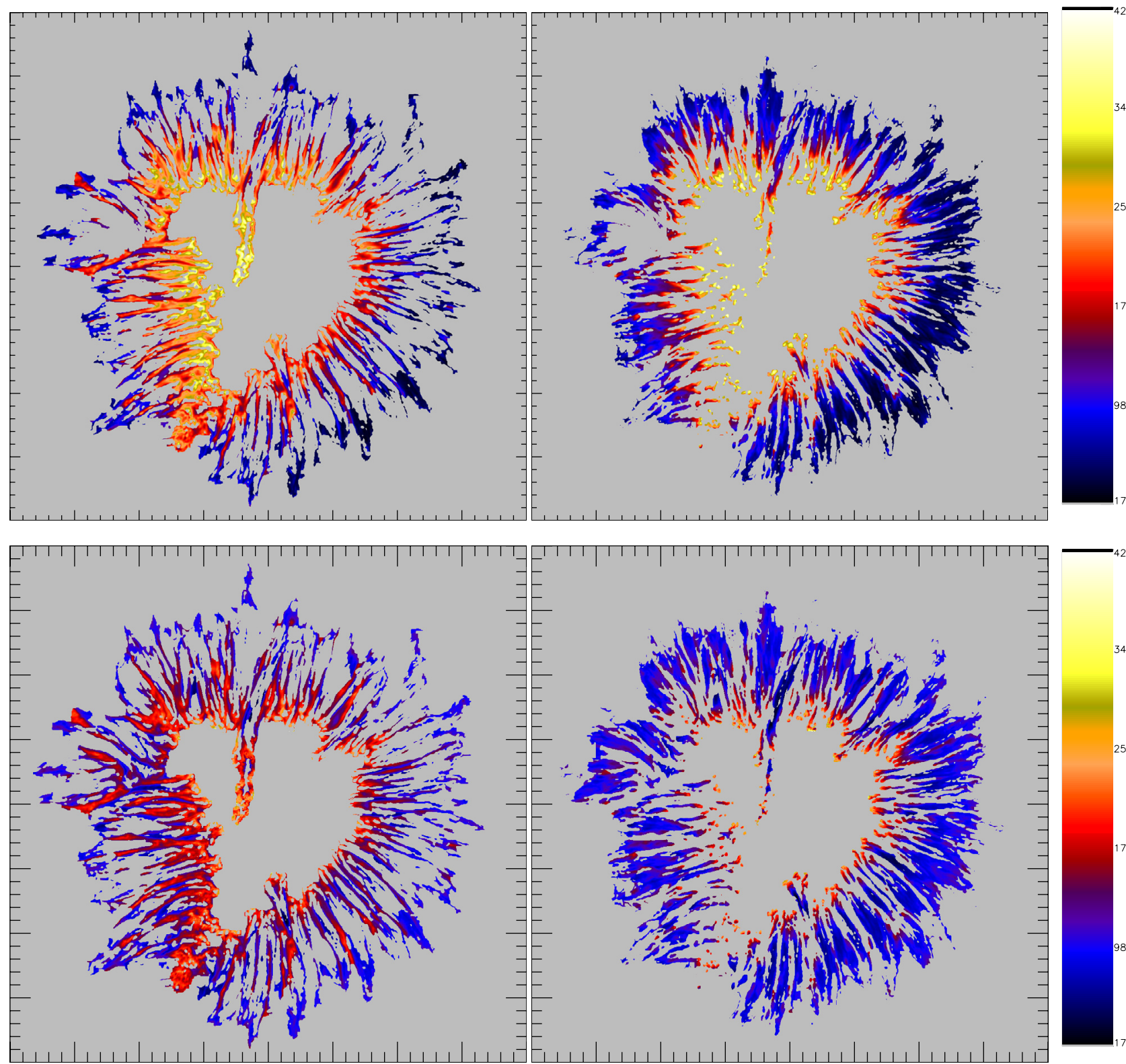

Fig. 3. Colour maps of TCP in the penumbra. Left column: pixels where the vertical temperature gradient is low. Right column: pixels where the vertical temperature gradient is high. Top row: no filtering. Bottom row: divided by continuum intensity then removed low pass component. Quantities in both images are scaled the same way. The colour scale is linear and in arbitrary units. Minor tickmark spacing is $1^{\prime \prime}$. Field of view is $40 \times 40^{\prime \prime}$.

(Scharmer et al. 2002) as seen in the intensity maps. The counterparts of these filaments in both TCP and gradient maps are mostly, but not always, dark features, which indicates both the absence of strong vertical fields (and thus necessarily a more horizontal component) and strong temperature gradients. The dark cores themselves are prominent in the TCP map, showing strongly reduced TCP, as was first noted by Bellot Rubio et al. (2007) in Hinode observations (also noted by Langhans et al. 2007 in circular polarisation in a single wavelength). The dark cores are less prominent in the gradient maps even though they generally appear dark there, implying stronger vertical temperature gradients than their surroundings. Thus, although complex, the relation between TCP and gradient in the penumbra is similar to that of the plage region of Fig. 2 (which includes pores, bright points, and granulation).

Figure 5 shows a portion of the centre-side inner penumbra and a light bridge. Here the dark-cored filaments stand out much more clearly than on the limb side, as first noted by Sütterlin et al. (2004). The dark cores are dark in all intensity maps shown, confirming the observation of Langhans et al. (2007) that they are discernible well above continuum optical depth of 0.1 . They are also dark in all the other maps, indicating that they are features with weak and/or horizontal magnetic fields and steep vertical temperature gradients. The dark lanes in the light bridge behave in exactly the same way.

Inspecting the sequence of intensity maps, we note that the dark cores, as well as the dark lanes of the light bridge, are wider and more pronounced in FW than in WB. In TF2 they are arguably thinner again, but even more contiguous. We thus see a tendency of the dark cores to look longer and more contiguous as we go from lower layers (WB) to higher (FW and on to TF2). The visual impression is that we sometimes "see below" the dark core in the continuum. The arrow points to a dark-cored filament that is a nice illustration of this. 


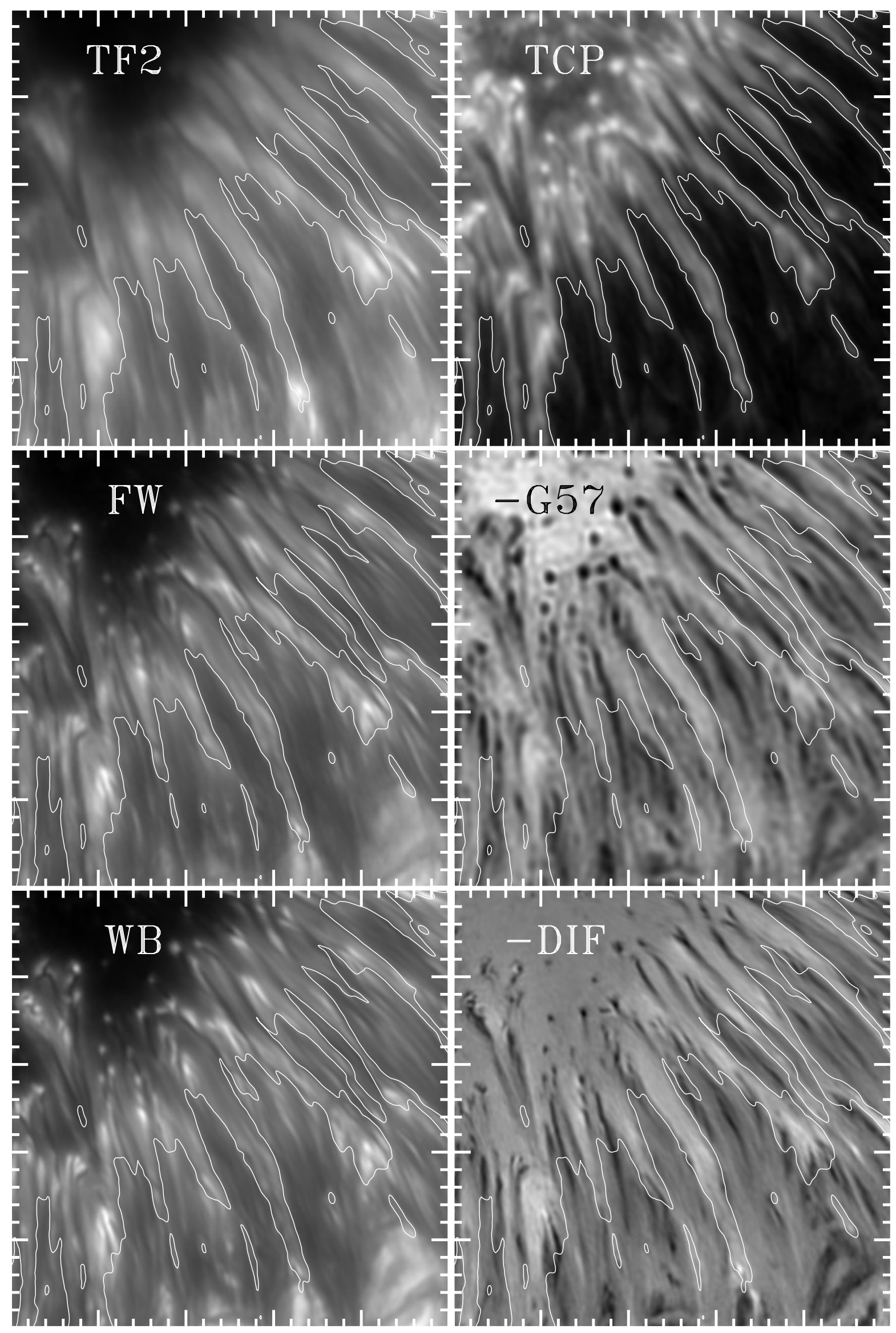

Fig. 4. A subfield from the limb-side penumbra. The contour is drawn for a TCP value chosen to outline the spine structure. Major tickmark spacing is $2^{\prime \prime}$. Field of view is $10 \times 10^{\prime \prime}$. 
V. M. J. Henriques and D. Kiselman: Ca II H sunspot tomography from the photosphere to the chromosphere

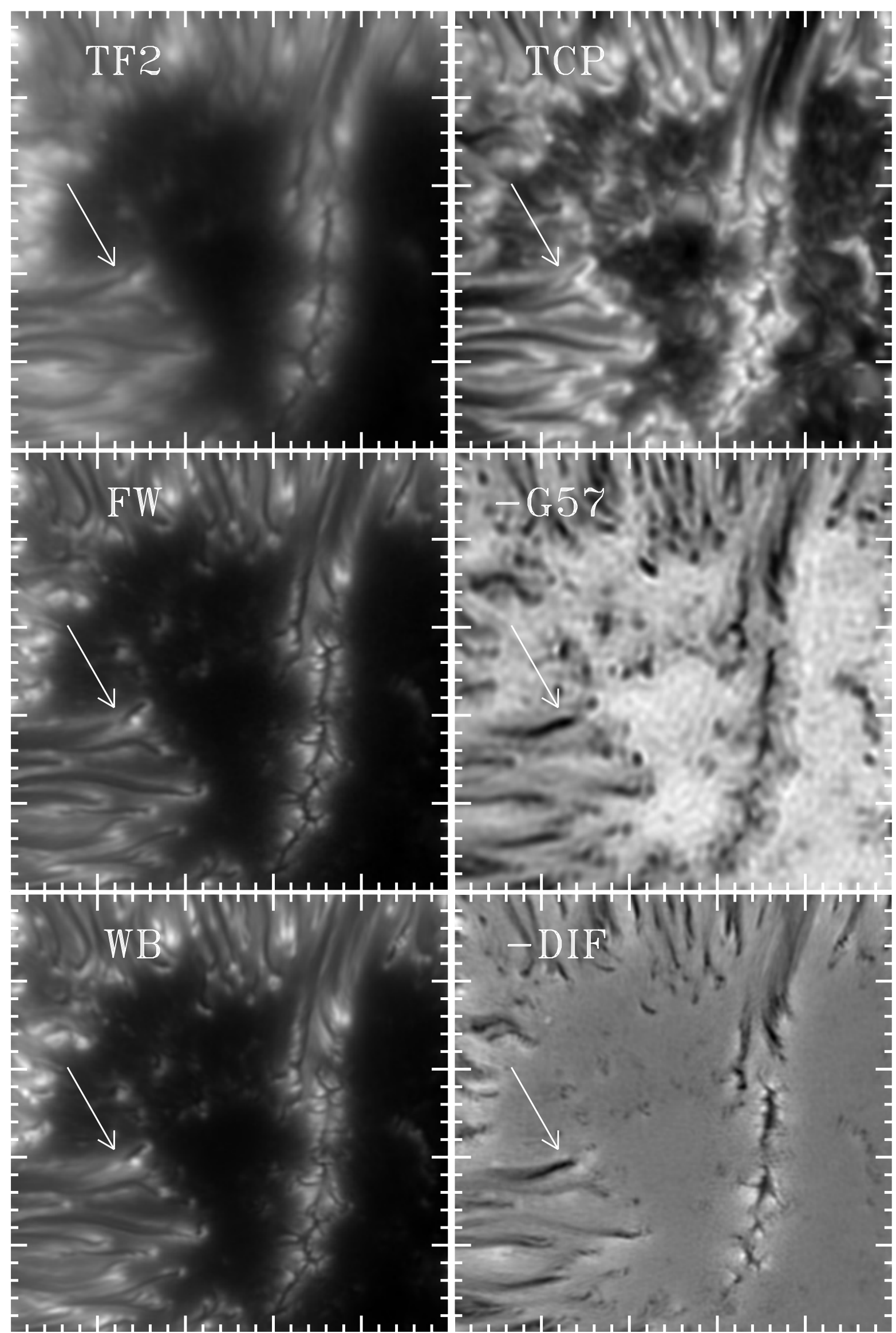

Fig. 5. Like Fig. 4 for a subfield at the umbral-penumbral border on the centre-side of the spot. Major tickmark spacing is $2^{\prime \prime}$. Field of view is $10 \times 10^{\prime \prime}$. 


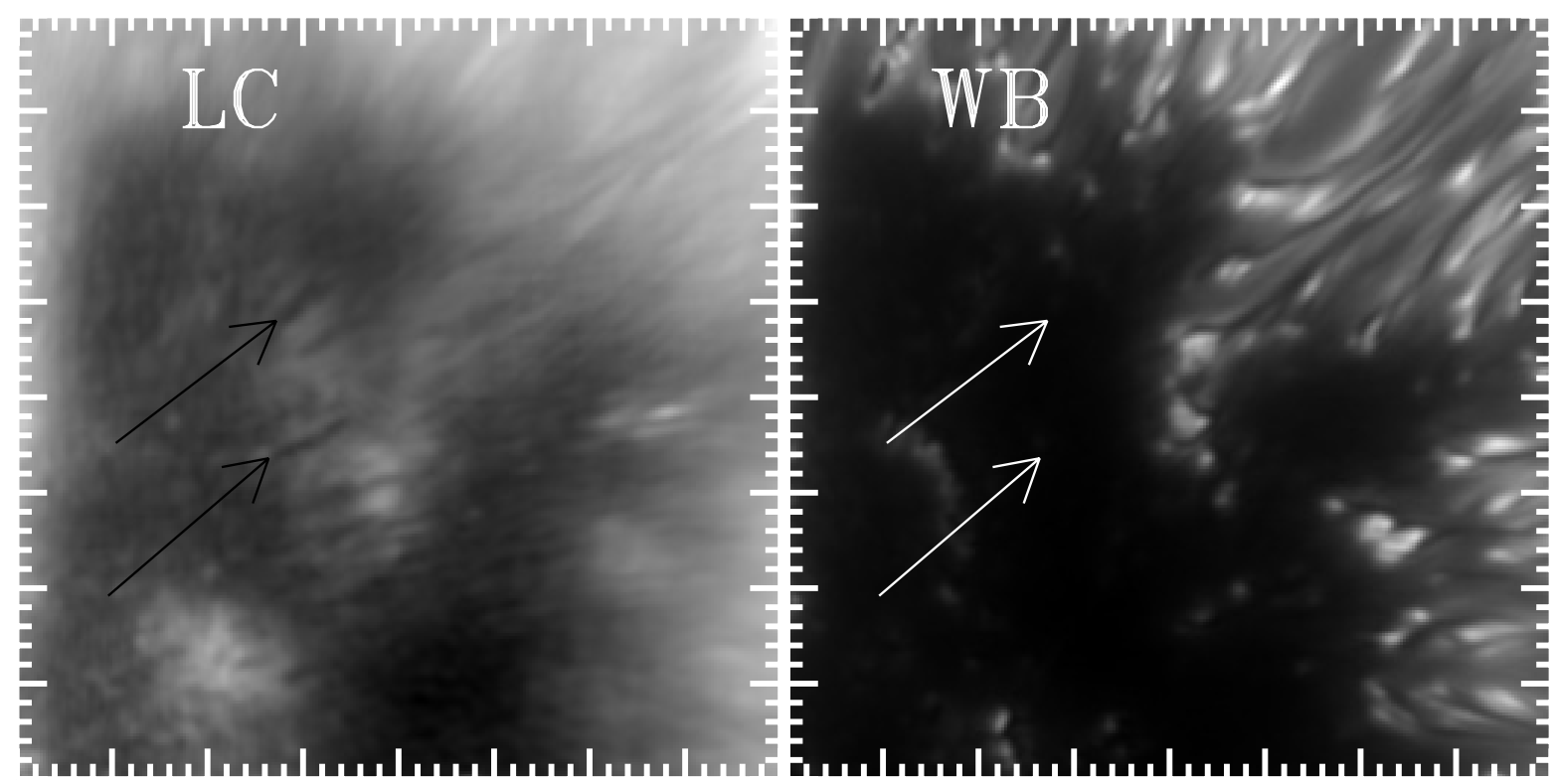

Fig. 6. Left: LC image of the umbra in logarithmic intensity scale. Right: WB. The arrows point at extended dark fibril-like structures as visible in LC. The coordinates of the arrows are the same in both images. Each major tickmark corresponds to $1^{\prime \prime}$. Field of view is $8 \times 8^{\prime \prime}$.

Finally, we note that umbral dots also show up in both gradient maps in the same way as the peripheral penumbral grains (filament heads) and thus have steep temperature gradients. This is however a case where the G57 and the DIF maps look different, with the umbral dots being much more pronounced in the former.

\section{Upper photosphere to chromosphere}

The LC images allow an unusually high-resolution look at the upper photosphere and chromosphere of the sunspot. While the filter width gives strong contamination from the inner wing of the $\mathrm{H}$ line, which is formed in the photosphere, fibril structures that must be of chromospheric origin are clearly visible. Pietarila et al. (2009) investigated these fibrils with filtergrams in the $\mathrm{K}$ line. They estimated that $90 \%$ of the intensity in their images was of photospheric origin. Their filter was, however, wider than the current one (1.5 ̊ compared to $1.1 \AA$ ), so we expect the present LC filtergram to show somewhat cleaner chromospheric features. Here we discuss briefly some of the features seen in order to illustrate the potential of the data.

That fibrils are visible also over a sunspot umbra was first reported by Socas-Navarro et al. (2009) from Hinode imagery. Figure 6 shows filamentary features over the current umbra. We note that to make these fibrils appear clearly in the image, a logarithmic intensity scaling has been used. The fibrils indicated with arrows are dark against a brighter background caused by an umbral flash. There are, however, also traces of bright fibrils against dark umbra. The width of the dark fibrils indicated by the arrows is about 0 '. 15 . This leads us to think that some of these structures may be resolved in the images. They cannot be an artefact from the reconstruction as dark fibrils can be seen on several consecutive frames but with the current data we are unable to say if exactly the same structures remain over multiple flashes. At least similar structures are visible during several flashes. Their maximum observed apparent length is nearly $5^{\prime \prime}$ or $3700 \mathrm{~km}$. This is somewhat larger than the $2000 \mathrm{~km}$ reported in Socas-Navarro et al. (2009). Also, for the same reason pointed out in that work (the broad but still limited formation height of the Ca II H linecore), a large inclination from the vertical is implied for these structures. We do not exclude the possibility that these structures are formed in the upper photosphere.

Figure 7 shows the same penumbral field as Fig. 4. Here too, many of the fibrils give the impression of being dark narrow features seen in absorption against a bright background. In some cases, like the one where a cross section is shown as a plot and others marked with arrows, one can speculate whether the dark central streak and associated bright features are part of the same magnetic structure and whether there is any connection to the dark-cored fibrils seen in Ca II IR lines and reported by Beck et al. (2010).

\section{Discussion and conclusion}

We summarise the observational results as follows.

The outer parts of the sunspot penumbra show a relation between vertical magnetic field (line-of-sight TCP) and vertical temperature gradients similar to that found outside the sunspot: magnetic regions are associated with relatively flat vertical temperature gradients. This relation does not hold at the umbral/penumbral border, where most penumbral structure shows high TCP values.

The penumbral intensity also correlates with TCP, so that spines have higher intensity in our maps, similar to what was found by Scharmer et al. (2011).

We confirm, at higher spatial resolution, the finding of Bellot Rubio et al. (2007) that dark-cored filaments are prominent in TCP maps. Furthermore, we show that they are also visible in temperature-gradient maps, associating the dark cores themselves with strong vertical temperature gradients and weaker and/or more horizontal magnetic fields than the surrounding penumbra.

The dark lanes in a light bridge behave in the same way as the dark cores of penumbral filaments in our maps. This is another indication that the two phenomena have a similar origin (Scharmer et al. 2007; Rimmele 2008). Following dark-core features in successively higher formed intensity passbands, we note that they look increasingly longer and more contiguous.

We also note that umbral dots in the gradient maps behave similarly to the peripheral penumbral grains. We claim that this 
V. M. J. Henriques and D. Kiselman: Ca II H sunspot tomography from the photosphere to the chromosphere
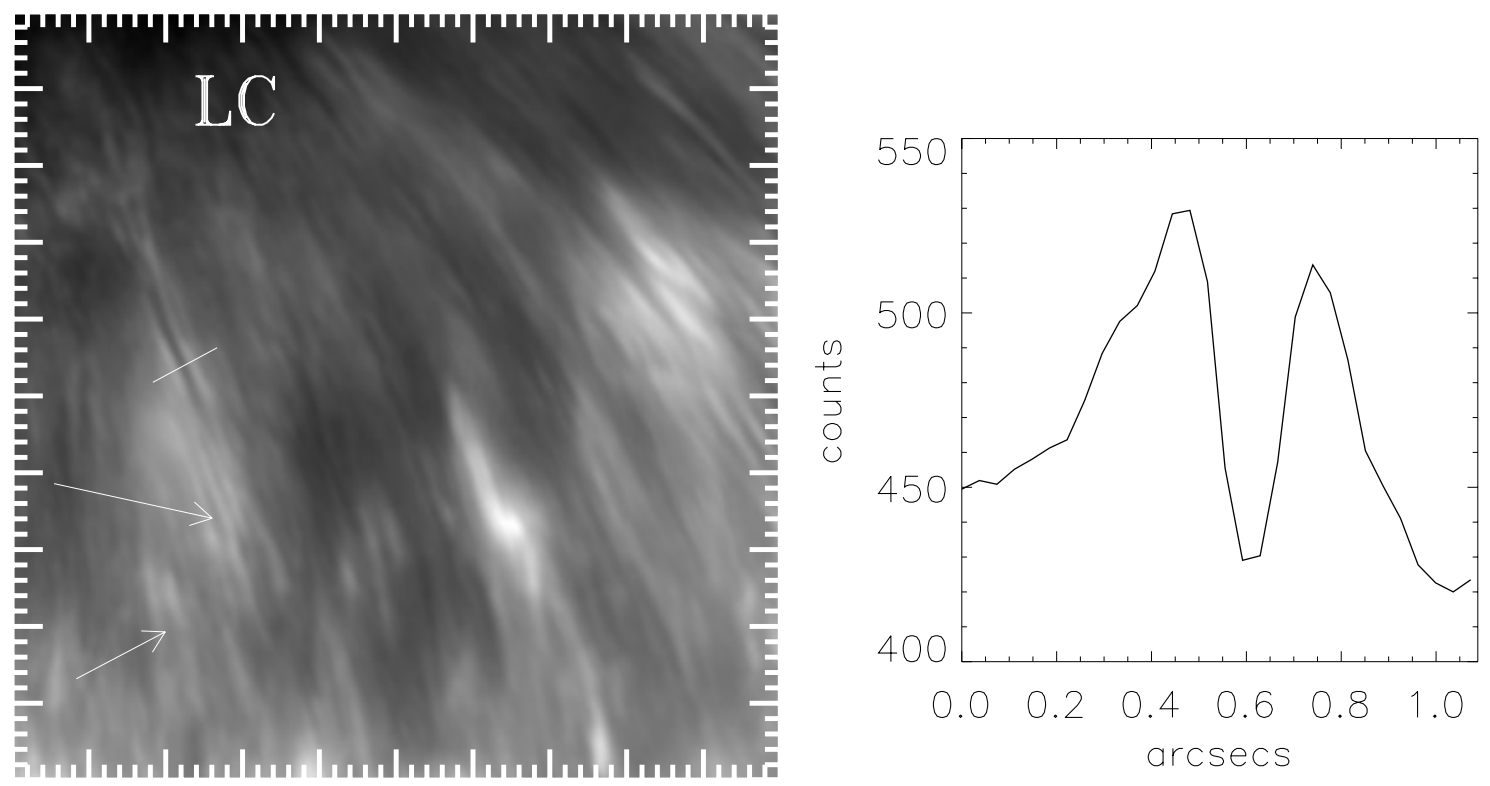

Fig. 7. Left: LC image of the same field as Fig. 4. The arrows point at extended dark fibril-like structures. Each major tickmark corresponds to 1". Right: intensity plot from the cut transversal to a dark feature drawn on the left panel.

gives independent support to the idea that penumbral filaments, light bridges, and umbral dots are essentially similar phenomena caused by overturning convection.

In our Ca II H line-core images, we confirm, at higher spatial resolution, the observations of fibril structure over the sunspot umbra of Socas-Navarro et al. (2009). Narrow fibrils are visible as dark streaks over bright regions due to umbral flashes, and similar structures are seen over the penumbra.

We conclude by noting that imaging in the $\mathrm{Ca}$ II $\mathrm{H}$ wing can produce data of the highest spatial resolution currently possible. With the exception of some regions in the sunspot umbra, we find that the methods from Henriques (2012) for co-aligning the different $\mathrm{Ca}$ images, as well as co-aligning these with data from other wavelengths, work well. The temperature-extraction procedures of Henriques (2012) are robust and seem to work well in the sunspot penumbra. The resulting temperature-gradient diagnostic gives an interesting angle on penumbral structure, and it would be most interesting to compare the observational results directly with simulations (such as those of Heinemann et al. 2007 and Rempel 2011) and with those obtained from inversions applied to narrow spectral lines. We also note the potential of using the temperatures derived from the $\mathrm{H}$ wing to decrease the number of free parameters in spectro-polarimetric inversions of observed profiles of magnetically sensitive lines.

Acknowledgements. This project was supported by a Marie Curie Early Stage Research Training Fellowship of the European Community's Sixth Framework Programme under contract number MEST-CT-2005-020395: The USO-SP International School for Solar Physics. Support was also received from H.M. Konungens Wallenbergsfond. The Swedish 1-m Solar Telescope is operated on La Palma by the Institute for Solar Physics of the Royal Swedish Academy of Sciences in the Spanish Observatorio del Roque de los Muchachos of the Instituto de Astrofísica de Canarias.

\section{References}

Beck, C., Tritschler, A., \& Wöger, F. 2010, Astron. Nachr., 331, 574 Beck, C., Rezaei, R., \& Puschmann, K. G. 2013, A\&A, 549, A24
Bellot Rubio, L. R., Tsuneta, S., Ichimoto, K., et al. 2007, ApJ, 668, L91

Centeno, R., Socas-Navarro, H., Collados, M., \& Trujillo Bueno, J. 2005, ApJ, 635,670

de la Cruz Rodríguez, J., Rouppe van der Voort, L., Socas-Navarro, H., \& van Noort, M. 2013, A\&A, 556, A115

Heinemann, T., Nordlund, Å., Scharmer, G. B., \& Spruit, H. C. 2007, ApJ, 669, 1390

Henriques, V. M. J. 2012, A\&A, 548, A114

Langhans, K., Scharmer, G. B., Kiselman, D., \& Löfdahl, M. G. 2007, A\&A, 464,763

Leenaarts, J., Rutten, R. J., Carlsson, M., \& Uitenbroek, H. 2006, A\&A, 452, L15

Lites, B. W., Elmore, D. F., Seagraves, P., \& Skumanich, A. P. 1993, ApJ, 418 928

Löfdahl, M. G., Henriques, V. M. J., \& Kiselman, D. 2011, A\&A, 533, A82

Pietarila, A., Hirzberger, J., Zakharov, V., \& Solanki, S. K. 2009, A\&A, 502, 647

Rempel, M. 2011, ApJ, 740, 15

Rimmele, T. 2008, ApJ, 672, 684

Rouppe van der Voort, L. H. M. 2002, A\&A, 389, 1020

Scharmer, G. B. 2006, A\&A, 447, 1111

Scharmer, G. B., \& Henriques, V. M. J. 2012, A\&A, 540, A19

Scharmer, G. B., Gudiksen, B. V., Kiselman, D., Löfdahl, M. G., \& Rouppe van der Voort, L. H. M. 2002, Nature, 420, 151

Scharmer, G. B., Bjelksjo, K., Korhonen, T. K., Lindberg, B., \& Petterson, B 2003, in SPIE Conf. Ser. 4853, eds. S. L. Keil, \& S. V. Avakyan, 341

Scharmer, G. B., Langhans, K., Kiselman, D., \& Löfdahl, M. G. 2007, in New Solar Physics with Solar-B Mission, eds. K. Shibata, S. Nagata, \& T. Sakurai, ASP Conf. Ser., 369, 71

Scharmer, G. B., Narayan, G., Hillberg, T., et al. 2008, ApJ, 689, L69

Scharmer, G. B., Henriques, V. M. J., Kiselman, D., \& d. 1. C. Rodríguez, J. 2011, Science, 333, 316

Selbing, J. 2010, Master thesis Stockholm University [arXiv: 1010.4142]

Sheminova, V. A. 2012, Sol. Phys., 280, 83

Sheminova, V. A., Rutten, R. J., \& Rouppe van der Voort, L. H. M. 2005, A\&A, 437, 1069

Shine, R. A., \& Linsky, J. L. 1974, Sol. Phys., 37, 145

Shine, R. A., Title, A. M., Tarbell, T. D., et al. 1994, ApJ, 430, 413

Socas-Navarro, H., Trujillo Bueno, J., \& Ruiz Cobo, B. 2000, Science, 288, 1398 Socas-Navarro, H., McIntosh, S. W., Centeno, R., de Wijn, A. G., \& Lites, B. W. 2009, ApJ, 696, 1683

Sütterlin, P., Bellot Rubio, L. R., \& Schlichenmaier, R. 2004, A\&A, 424, 1049

Tritschler, A., Uitenbroek, H., \& Reardon, K. 2008, ApJ, 686, L45

van Noort, M., Rouppe van der Voort, L., \& Löfdahl, M. G. 2005, Sol. Phys., 228,191

Vernazza, J. E., Avrett, E. H., \& Loeser, R. 1981, ApJS, 45, 635 